\title{
Potential Effects of Biochar Application for Improving Wheat (Triticum aestivum L.) Growth and Soil Biochemical Properties under Drought Stress Conditions
}

\author{
Muhammad Saqlain Zaheer ${ }^{1}\left(\mathbb{D}\right.$, Hafiz Haider Ali ${ }^{2}$, Walid Soufan ${ }^{3}{ }^{(\mathbb{D}}$, Rashid Iqbal ${ }^{4}(\mathbb{D}$, \\ Muhammad Habib-ur-Rahman ${ }^{5,6}{ }^{\circ}$, Javaid Iqbal ${ }^{7}{ }^{(0)}$, Muhammad Israr ${ }^{8}{ }^{-1}$ and Ayman El Sabagh ${ }^{9, *}$
}

1 Department of Agricultural Engineering, Khwaja Fareed University of Engineering and Information Technology, Rahim Yar Khan 64200, Pakistan; saqlain.zaheer@kfueit.edu.pk

2 Sustainable Development Study Center (SDSC), Government College University, Katchery Road, Lahore 54000, Pakistan; dr.haiderali@gcu.edu.pk

3 Plant Production Department, College of Food and Agriculture Sciences, King Saud University, P.O. Box 2460, Riyadh 11451, Saudi Arabia; wsoufan@ksu.edu.sa

4 Department of Agronomy, The Islamia University of Bahawalpur, Bahawalpur 63100, Pakistan; rashid.iqbal@iub.edu.pk

5 Crop Science, Institute of Crop Science and Resource Conservation (INRES), University of Bonn, 53115 Bonn, Germany; habib.rahman@mnsuam.edu.pk

check for updates

Citation: Zaheer, M.S.; Ali, H.H.; Soufan, W.; Iqbal, R.;

Habib-ur-Rahman, M.; Iqbal, J.; Israr, M.; El Sabagh, A. Potential Effects of Biochar Application for Improving Wheat (Triticum aestivum

L.) Growth and Soil Biochemical Properties under Drought Stress Conditions. Land 2021, 10, 1125. https://doi.org/10.3390/ land10111125

Academic Editors: Valerie Vranová, Rahul Datta and Subhan Danish

Received: 11 September 2021

Accepted: 19 October 2021

Published: 22 October 2021

Publisher's Note: MDPI stays neutral with regard to jurisdictional claims in published maps and institutional affiliations.

Copyright: () 2021 by the authors. Licensee MDPI, Basel, Switzerland. This article is an open access article distributed under the terms and conditions of the Creative Commons Attribution (CC BY) license (https:// creativecommons.org/licenses/by/ $4.0 /)$.
6 Department of Agronomy, MNS-University of Agriculture Multan, Multan 59210, Pakistan

7 Department of Plant Protection, College of Food and Agriculture Sciences, King Saud University, P.O. Box 2460, Riyadh 11451, Saudi Arabia; jiqbal@ksu.edu.sa

8 Department of Biology, The University of Haripur, Haripur 22620, Pakistan; m.israr@uoh.edu.pk

9 Department of Agronomy, Faculty of Agriculture, Kafrelsheikh University, Kafr El-Shaikh 33516, Egypt

* Correspondence: ayman.elsabagh@agr.kfs.edu.eg

\begin{abstract}
Different soil amendments are applied to improve soil properties and to achieve higher crop yield under drought conditions. The objective of the study was to investigate the role of biochar for the improvement of wheat (Triticum aestivum L.) growth and soil biochemical properties under drought conditions. A pot experiment with a completely randomized design was arranged with four replications in a wire house. Drought was imposed on two critical growth stages (tillering and grain filling) and biochar was applied to the soil 10 days before sowing at two different rates $\left(28 \mathrm{~g} \mathrm{~kg}^{-1}\right.$ and $38 \mathrm{~g} \mathrm{~kg}^{-1}$ ). Soil samples were collected to determine the soil properties including soil respiration and enzymatic parameters after crop harvesting. Results showed that water stress negatively affects all biochemical properties of the soil, while biochar amendments positively improved these properties. Application of biochar at $38 \mathrm{~g} \mathrm{~kg}^{-1}$ provided significantly higher mineral nutrients, Bray $\mathrm{P}(18.72 \%)$, exchangeable-K $(7.44 \%)$, soil carbon $(11.86 \%)$, nitrogen mineralization $(16.35 \%)$, and soil respiration $(6.37 \%)$ as a result of increased microbial activities in comparison with the $28 \mathrm{~g} \mathrm{~kg}^{-1}$ rate.
\end{abstract}

Keywords: biochar; drought; enzymatic activities; mineral nutrients; soil properties

\section{Introduction}

Wheat (Triticum aestivum L.) is the most demanding crop and the second most important staple food crop after maize in the world. It also plays a vital role in the economy of the agriculture sector [1,2]. Drought is the most damaging abiotic stress responsible for severe yield reduction in wheat by imposing detrimental effects on seed germination, seedling development, and overall crop physiology [3,4]. Climate variability from the last few years leads towards the increase of average temperature and drier summer, especially in Pakistan. More demand for water and less availability increases the drought risk in many areas of the world. Drought stress is a main constrain in sustainable wheat production [2,4].

Increase in the atmospheric $\mathrm{CO}_{2}$ results in the change of the climate and also changing of the rainfall pattern and seasonal drought conditions [5]. Drought is becoming a serious 
issue for crop production in changing climatic conditions so there is a need to manage the crop production accordingly [4-6]. Drought stress can damage the root growth of the wheat plant in the initial stages of plant growth germination. Poor hydraulic conductance in the leaves and the root can alter the whole plant's physiological and metabolic functions. Global warming and food insecurity are forcing farmers to enhance crop production under limited water conditions but it is very difficult to achieve under the current change in climatic scenario [6]. Drought stress can also damage the nutrient uptake by the plant and also disturb the fertilizer application for crop production as some fertilizers are volatile and not available to the plant under dry conditions. Sowing and crop management, including all agricultural practices, can affect drought stress [4].

Soil management with the optimum utilization of the resources by which farmers can sustain the soil productivity is essential in current intensive agriculture. High use of chemical fertilizers, pesticides, and other chemicals in crop production cause a decline in soil fertility that is also damaging the chemical and physical properties of the soil [7]. Microbial activity and the decomposition of the various inorganic materials are damaging due to the current use of chemicals $[7,8]$. There is a need to manage soil with the use of organic matter by which we can retain soil fertility [9]. Soil quality plays a vital role in higher crop production and its evaluation under drought conditions can identify the problematic area for sustainable production and soil productivity [8]. Soil productivity refers to the favorable environment for plant growth that has suitable nutrients and growth medium. It also links with the soil quality and it can be determined by different indicators such as chemical, biological, and physical properties. Good soil quality can produce better crops [9]. The cation exchange capacity is also an indicator to investigate the soil texture. Soil texture and structure are also dependent on the soil's biochemical properties [10].

Improvement in soil properties with the application of organic amendments has been suggested as a way to enhance productivity and also to mitigate the detrimental impacts of drought [11]. Biochar, a pyrogenic carbon that is commonly derived from the combustion of carbon-rich materials, has recently attracted considerable attention both as a soil conditioner and as a buffer against drought stress [12]. The biochar addition to the soil has been reported to increase the soil organic carbon, moisture contents, and porosity, increase cation exchange capacity, as well as increase retention of polyvalent cations $[10,13]$. Biochar application in soil was reported by Baiamonte et al. [14] and Odugbenro et al. [15] to increase the stability of soil texture and aggregates. Soil water retention can also be effective by the aggregate stability, particularly under low soil water content [16]. However, the water-retaining ability of biochar amended soils may vary with the soil type where it is applied, the source and rate of biochar application, as well as with the biochar preparation methods $[17,18]$.

It is generally known that biochar application improves the soil properties under limited water conditions under semiarid conditions $[12,18]$. However, limited studies exist investigating the biochar application under limited water stress conditions. Therefore, the present study aimed to elucidate the effect of biochar application on soil chemical properties and wheat growth under drought stress conditions. We hypothesized that the biochar addition in the soil as a soil amendment will improve the soil properties, water-related parameters, and growth of a water-stressed wheat plant.

\section{Materials and Methods}

\subsection{Experimental Site and Design}

The experiment was conducted from 2018 to 2019 at the wire house of the Islamia University Bahawalpur, Pakistan. Cultivar "Galaxy-2013", an approved wheat seed variety from Punjab Seed Corporation, was used in the experiment. Six wheat seeds were sown into pots $(2 \times 2 \times 2$ feet $)$ containing $35 \mathrm{~kg}$ of soil maintained. Soil analysis was also done before the experiment, the soil was contained $0.74 \%$ organic matter, $29 \mathrm{mg} \mathrm{kg}^{-1}$ available-P and $75 \mathrm{mg} \mathrm{kg}^{-1}$ available- $\mathrm{K}, 231 \mu \mathrm{S} / \mathrm{cm}$ electrical conductivity, $0.54 \%$ total soluble salts, 
and $36 \%, 40 \%, 24 \%$ sand, silt, clay contents, respectively. The average temperature and humidity during the experiment are given in Table 1.

Table 1. Average temperature and humidity during the crop growth period.

\begin{tabular}{ccc}
\hline Month & Temperature $\left({ }^{\circ} \mathbf{C}\right)$ & Humidity (\%) \\
\hline November & 20 & 61 \\
December & 14 & 70 \\
January & 13 & 73 \\
February & 15 & 68 \\
March & 21 & 61 \\
April & 29 & 48 \\
\hline
\end{tabular}

Biochar was used as soil amendments before 10 days of sowing wheat seeds. Biochar was obtained from the COMSATS Institute of Information Technology, Islamabad. Biochar used in the experiment was highly pyrolysis at a temperature of $700{ }^{\circ} \mathrm{C}$. Biochar was applied with different doses according to the treatments in pots (without biochar $\left(\mathrm{B}_{0}\right)$, with biochar applied at $28 \mathrm{~g} \mathrm{~kg}^{-1}\left(\mathrm{~B}_{1}\right), 38 \mathrm{~g} \mathrm{~kg}^{-1}\left(\mathrm{~B}_{2}\right)$. Drought was imposed at tillering stage $\left(D_{1}\right)$ or grain filling stage $\left(D_{2}\right)$ of crop growth by completely withdrawing irrigation. Fully irrigated $\left(D_{0}\right)$ crops were treated as control. Irrigation was applied at critical growth stages and 3 inch pots were filled with water during the irrigation. Characteristics of used biochar in the experiment are given in Table 2. All other inputs remain the same according to the recommended dose. Planting pots were settled in a complete randomized (CRD) design with four replications. The total planting period was 180 days.

Table 2. Properties of biochar applied.

\begin{tabular}{lc}
\hline \multicolumn{1}{c}{ Biochar Property } & Values \\
\hline $\mathrm{pH}$ & 10.1 \\
Electric conductivity $\left(\mu \mathrm{S} \mathrm{cm}^{-1}\right)$ & 938 \\
$\mathrm{TC}(\%)$ & 67.2 \\
$\mathrm{TN}(\%)$ & 0.65 \\
Dichromate oxidizable organic C & 4.54 \\
Water Soluble Carbon & 152 \\
Water Soluble Nitrogen & 94.23 \\
Ash content $(\%)$ & 3.31 \\
Carbonates content $\left(\% \mathrm{CaCO}_{3}\right)$ & 11.45 \\
$\left.\mathrm{CEC} \mathrm{(cmol} \mathrm{kg}^{-1}\right)$ & 34.21 \\
$\mathrm{NH}_{4}-\mathrm{N}$ sorption $\left(\mathrm{mg} \mathrm{NH}_{4}-\mathrm{N} \mathrm{g} \mathrm{g}^{-1}\right)$ & 2.12 \\
$\mathrm{NO}_{3}-\mathrm{N}$ sorption $\left(\mathrm{mg} \mathrm{NO}_{3}-\mathrm{N} \mathrm{g}^{-1}\right)$ & $\mathrm{N} . \mathrm{S}$. \\
\hline
\end{tabular}

\subsection{Soil Sampling}

Soil samples were taken from the pots at different growth stages of the wheat crop and stored in plastic bags before transporting to the laboratory. Additional pots were already arranged to take the multiple soil samples from each growth stage that had the same applications and growth conditions. Sampling was done at two different phonological stages of wheat: tillering and grain filling. For that, different pots were prepared for soil sampling at tillering and grain filling periods having the same conditions. After shifting of the soil samples in the lab, it was sieved $(<2 \mathrm{~mm})$ and analyzed for soil moisture content. After sieving, the soil was stored at $4{ }^{\circ} \mathrm{C}$.

Bray $\mathrm{P}, \mathrm{SOC}$, and exchangeable $\mathrm{K}$ were determined with standard methods demonstrated by Sparks et al. $[19,20]$. Soil pH was determined in a soil and water suspension. Total $\mathrm{N}$ was determined by steam distillation as reported by Mulvaney [21]. Dissolved organic nitrogen (DON) and carbon (DOC) were measured according to the procedure reported by Smolander and Kitunen [22]. Nitrogen mineralization rate (NMIN) was calculated from the soil before and after 10 days of incubation at $30^{\circ} \mathrm{C}$. Soil microbial biomass $\mathrm{C}, \mathrm{N}$, and 
P were measured by using fumigation extraction methods as reported by Vance et al. [23] and Heinze et al. [24]. Soil Respiration (SR) was determined according to Dinesh et al. [20]. Soil enzyme activities such as urease (UR), dehydrogenase (DH), $\beta$-glucosidase ( $\beta G$ ), and acid phosphatase (AcP) activities in the soil were determined by the procedure described by Tabatabai [25].

\subsection{Statistical Analysis}

Data were analyzed by using the Fisher's test for analysis of variance with fixed factors biochar amendment (unamended, $28 \mathrm{~g} \mathrm{~kg}^{-1}$ and $38 \mathrm{~g} \mathrm{~kg}^{-1}$ ) and moisture treatment (constantly irrigated, a drought at tillering and grain filling stages). The least significant difference (LSD) was used to determine significant differences for the amendment $\times$ moisture treatment interaction at 95\% confidence level $(p \leq 0.05)$ [26].

\section{Results}

Growth and yield-related parameters of the wheat crop were significantly affected by the drought and it was also noticed that biochar application has a significant effect to enhance the growth and yield of wheat (Table 3). The highest values were noticed with the application of biochar at a rate of $38 \mathrm{~g} \mathrm{~kg}^{-1}$ and the lowest was found when there was no application of biochar. It was also found that the lowest plant height, spike length, and the number of spikelets were noticed when drought was applied at the tillering stage but a minimum number of grains, 1000-grain weight, and grain yield were observed when drought stress was applied at the grain filling stage. The same kind of trends in the results was noticed in the interaction with the biochar and drought stress was noticed.

Table 3. Effect of biochar on growth and yield-related parameters of wheat crop under water-limited stress condition.

\begin{tabular}{|c|c|c|c|c|c|c|}
\hline Treatments & $\begin{array}{l}\text { Plant Height } \\
\text { (cm) }\end{array}$ & $\begin{array}{l}\text { Spike Length } \\
\text { (cm) }\end{array}$ & $\begin{array}{l}\text { Number of } \\
\text { Spikelets per } \\
\text { Spike }\end{array}$ & $\begin{array}{l}\text { Number of } \\
\text { Grains per } \\
\text { Spike }\end{array}$ & $\begin{array}{l}\text { 1000-Grain } \\
\text { Weight (g) }\end{array}$ & $\begin{array}{l}\text { Grain Yield } \\
\text { per Plant (g) }\end{array}$ \\
\hline $\mathrm{D}_{0}$ & $97 \mathrm{~A}( \pm 0.912)$ & $10.45 \mathrm{~A}( \pm 0.144)$ & $24.33 \mathrm{~A}( \pm 0.120)$ & $46 \mathrm{~A}( \pm 0.353)$ & $35 \mathrm{~A}( \pm 0.359)$ & $1.48 \mathrm{~A}( \pm 0.009)$ \\
\hline $\mathrm{D}_{1}$ & 87 C $( \pm 0.938)$ & $08.88 \mathrm{C}( \pm 0.074)$ & $22.24 \mathrm{~B}( \pm 0.087)$ & $40 \mathrm{~B}( \pm 0.678)$ & 32 B $( \pm 0.359)$ & $0.90 \mathrm{~B}( \pm 0.015)$ \\
\hline $\mathrm{D}_{2}$ & $93 \mathrm{~B}( \pm 0.408)$ & $09.55 \mathrm{~B}( \pm 0.165)$ & $19.43 \mathrm{C}( \pm 0.038)$ & $37 \mathrm{C}( \pm 0.912)$ & $28 \mathrm{C}( \pm 0.156)$ & $0.50 \mathrm{C}( \pm 0.029)$ \\
\hline $\mathrm{B}_{0}$ & $90 \mathrm{C}( \pm 0.707)$ & $10.11 \mathrm{C}( \pm 0.047)$ & $21.24 \mathrm{C}( \pm 0.010)$ & $39 \mathrm{C}( \pm 0.496)$ & $30 \mathrm{C}( \pm 0.370)$ & $1.21 \mathrm{C}( \pm 0.013)$ \\
\hline $\mathrm{B}_{1}$ & $95 \mathrm{~B}( \pm 0.407)$ & $11.23 \mathrm{~B}( \pm 0.086)$ & $23.35 \mathrm{~B}( \pm 0.015)$ & $43 \mathrm{~B}( \pm 0.912)$ & 33 B $( \pm 0.389)$ & $1.34 \mathrm{~B}( \pm 0.017)$ \\
\hline $\mathrm{B}_{2}$ & $99 \mathrm{~A}( \pm 0.410)$ & $12.02 \mathrm{~A}( \pm 0.062)$ & $25.42 \mathrm{~A}( \pm 0.019)$ & $47 \mathrm{~A}( \pm 0.577)$ & $36 \mathrm{~A}( \pm 0.131)$ & $1.48 \mathrm{~A}( \pm 0.007)$ \\
\hline \multicolumn{7}{|l|}{$\mathrm{D} \times \mathrm{B}$} \\
\hline $\mathrm{D}_{0} \mathrm{~B}_{0}$ & 96.12 c $( \pm 1.037)$ & 10.27 c $( \pm 0.073)$ & 22.33 c $( \pm 0.043)$ & 43 c $( \pm 0.408)$ & 34.33 c $( \pm 0.134)$ & $1.25 \mathrm{c}( \pm 0.014)$ \\
\hline $\mathrm{D}_{0} \mathrm{~B}_{1}$ & $98.32 \mathrm{~b}( \pm 1.178)$ & $11.23 \mathrm{~b}( \pm 0.062)$ & $24.25 \mathrm{~b}( \pm 0.055)$ & $46 \mathrm{~b}( \pm 0.341)$ & $36.34 \mathrm{~b}( \pm 0.073)$ & $1.30 \mathrm{~b}( \pm 0.012)$ \\
\hline $\mathrm{D}_{0} \mathrm{~B}_{2}$ & 99.85 a $( \pm 0.828)$ & $12.02 \mathrm{a}( \pm 0.259)$ & 26.47 a $( \pm 0.090)$ & 48 a $( \pm 0.375)$ & 38.23 a $( \pm 0.112)$ & $1.46 \mathrm{a}( \pm 0.014)$ \\
\hline $\mathrm{D}_{1} \mathrm{~B}_{0}$ & $86.15 \mathrm{~h}( \pm 0.582)$ & 07.23 h $( \pm 0.089)$ & $19.13 \mathrm{f}( \pm 0.062)$ & $39 \mathrm{f}( \pm 0.408)$ & $30.43 \mathrm{f}( \pm 0.212)$ & $0.89 \mathrm{f}( \pm 0.004)$ \\
\hline $\mathrm{D}_{1} \mathrm{~B}_{1}$ & $88.53 \mathrm{~g}( \pm 1.167)$ & $08.25 \mathrm{~g}( \pm 0.179)$ & 20.54 e $( \pm 0.131)$ & 41 e $( \pm 0.177)$ & 31.34 e $( \pm 0.027)$ & 1.02 e $( \pm 0.007)$ \\
\hline $\mathrm{D}_{1} \mathrm{~B}_{2}$ & $90.23 \mathrm{f}( \pm 0.256)$ & 09.01 ef $( \pm 0.072)$ & $21.43 \mathrm{~d}( \pm 0.046)$ & $42 \mathrm{~d}( \pm 0.108)$ & $33.31 \mathrm{~d}( \pm 0.191)$ & $1.11 \mathrm{~d}( \pm 0.010)$ \\
\hline $\mathrm{D}_{2} \mathrm{~B}_{0}$ & 93.14 e $( \pm 0.402)$ & 09.12 de $( \pm 0.046)$ & $16.33 \mathrm{I}( \pm 0.032)$ & $30 \mathrm{I}( \pm 0.361)$ & $24.24 \mathrm{I}( \pm 0.088)$ & $0.56 \mathrm{I}( \pm 0.012)$ \\
\hline $\mathrm{D}_{2} \mathrm{~B}_{1}$ & $95.16 \mathrm{~d}( \pm 0.665)$ & $09.98 \mathrm{~d}( \pm 0.014)$ & $17.23 \mathrm{~h}( \pm 0.030)$ & $33 \mathrm{~h}( \pm 0.093)$ & $26.22 \mathrm{~h}( \pm 0.106)$ & $0.67 \mathrm{~h}( \pm 0.015)$ \\
\hline $\mathrm{D}_{2} \mathrm{~B}_{2}$ & 96.03 c $( \pm 0.724)$ & $10.24 \mathrm{c}( \pm 0.172)$ & $18.21 \mathrm{~g}( \pm 0.009)$ & $34 \mathrm{f}( \pm 0.410)$ & $28.32 \mathrm{~g}( \pm 0.080)$ & $0.80 \mathrm{~g}( \pm 0.009)$ \\
\hline
\end{tabular}

The different letter shows a significant difference in treatments at $5 \%$ probability level. $\mathrm{D}_{0}=$ Control, $\mathrm{D}_{1}=$ Drought at tillering stage, $\mathrm{D}_{2}=$ Drought at grain filling, $\mathrm{B}_{0}=($ No-Biochar $), \mathrm{B}_{1}=28 \mathrm{~g} \mathrm{~kg}^{-1}$ Biochar, $\mathrm{B}_{2}=38 \mathrm{~g} \mathrm{~kg}^{-1}$ Biochar. Values in brackets are standard error of the mean $(n=4)$.

Soil pH, total N, Exchangeable K, and Bray P were highest in soil under full irrigation with the highest biochar amendment but were lowest when drought was imposed at grain filling without biochar addition (Table 4). Under different soil moisture conditions (full irrigation or drought), high values of these soil parameters were found with the application of the highest biochar rate but were lowest in un-amended soil. Generally, with or without biochar amendment, these parameters were greatest in the soil of fully irrigated crops but were lowest under drought at the grain filling stage. Except that Bray P did not differ in biochar amended soil exposed to drought at both tillering and grain filling stages. 
Table 4. Effect of Biochar on Soil pH, Mineral N, Bray P and Exchangeable-K of under drought stress condition.

\begin{tabular}{|c|c|c|c|c|}
\hline Treatments & Soil pH & $\begin{array}{r}\text { Mineral N } \\
\left(\mathrm{mg} \mathrm{kg}^{-1}\right)\end{array}$ & Bray $P$ & Exchangeable-K \\
\hline $\mathrm{D}_{0}$ & $7.01 \mathrm{~A}( \pm 0.018)$ & $83.53 \mathrm{~A}( \pm 0.086)$ & $7.82 \mathrm{~A}( \pm 0.013)$ & $173.53 \mathrm{~A}( \pm 0.115)$ \\
\hline $\mathrm{D}_{1}$ & $6.85 \mathrm{~B}( \pm 0.088)$ & 80.17 B $( \pm 0.049)$ & $7.43 \mathrm{~B}( \pm 0.016)$ & $155.14 \mathrm{~B}( \pm 0.073)$ \\
\hline $\mathrm{D}_{2}$ & $6.78 \mathrm{C}( \pm 0.067)$ & $76.48 \mathrm{C}( \pm 0.015)$ & $6.98 \mathrm{C}( \pm 0.028)$ & $141.16 \mathrm{C}( \pm 0.083)$ \\
\hline $\mathrm{B}_{0}$ & $6.72 \mathrm{C}( \pm 0.061)$ & $66.81 \mathrm{C}( \pm 0.039)$ & $6.45 \mathrm{C}( \pm 0.209)$ & $105.79 \mathrm{C}( \pm 7.228)$ \\
\hline $\mathrm{B}_{1}$ & $6.87 \mathrm{~B}( \pm 0.070)$ & 84.53 B $( \pm 0.088)$ & $7.21 \mathrm{~B}( \pm 0.414)$ & $179.88 \mathrm{~B}( \pm 8.461)$ \\
\hline $\mathrm{B}_{2}$ & $7.05 \mathrm{~A}( \pm 0.079)$ & $88.84 \mathrm{~A}( \pm 0.047)$ & $8.56 \mathrm{~A}( \pm 0.128)$ & 193.17 A $( \pm 7.007)$ \\
\hline \multicolumn{5}{|l|}{$\mathrm{D} \times \mathrm{B}$} \\
\hline $\mathrm{D}_{0} \mathrm{~B}_{0}$ & $6.82 \mathrm{~d}( \pm 0.011)$ & $70.15 \mathrm{f}( \pm 0.092)$ & 6.82 e $( \pm 0.014)$ & $120.14 \mathrm{~g}( \pm 0.093)$ \\
\hline $\mathrm{D}_{0} \mathrm{~B}_{1}$ & $7.01 \mathrm{~b}( \pm 0.003)$ & $88.30 \mathrm{~b}( \pm 0.153)$ & 7.82 c $( \pm 0.014)$ & $196.26 \mathrm{~b}( \pm 0.135)$ \\
\hline $\mathrm{D}_{0} \mathrm{~B}_{2}$ & $7.23 \mathrm{a}( \pm 0.017)$ & 92.16 a $( \pm 0.080)$ & $8.82 \mathrm{a}( \pm 0.014)$ & 204.22 a $( \pm 0.124)$ \\
\hline $\mathrm{D}_{1} \mathrm{~B}_{0}$ & $6.72 \mathrm{e}( \pm 0.014)$ & $67.18 \mathrm{~g}( \pm 0.097)$ & $6.43 \mathrm{f}( \pm 0.023)$ & $100.11 \mathrm{~h}( \pm 0.066)$ \\
\hline $\mathrm{D}_{1} \mathrm{~B}_{1}$ & $6.81 \mathrm{~d}( \pm 0.009)$ & $85.18 \mathrm{~d}( \pm 0.096)$ & $7.41 \mathrm{~d}( \pm 0.008)$ & 170.18 e $( \pm 0.095)$ \\
\hline $\mathrm{D}_{1} \mathrm{~B}_{2}$ & $7.02 \mathrm{~b}( \pm 0.012)$ & 88.14 b $( \pm 0.093)$ & $8.44 \mathrm{~b}( \pm 0.023)$ & $195.11 \mathrm{c}( \pm 0.060)$ \\
\hline $\mathrm{D}_{2} \mathrm{~B}_{0}$ & $6.61 \mathrm{f}( \pm 0.008)$ & $63.11 \mathrm{~h}( \pm 0.061)$ & $6.10 \mathrm{f}( \pm 0.052)$ & $97.11 \mathrm{i}( \pm 0.066)$ \\
\hline $\mathrm{D}_{2} \mathrm{~B}_{1}$ & $6.81 \mathrm{~d}( \pm 0.007)$ & 80.11 e $( \pm 0.060)$ & 6.42 ef $( \pm 0.014)$ & $146.18 \mathrm{f}( \pm 0.092)$ \\
\hline $\mathrm{D}_{2} \mathrm{~B}_{2}$ & $6.92 \mathrm{c}( \pm 0.011)$ & 86.23 c $( \pm 0.115)$ & $8.43 \mathrm{~b}( \pm 0.020)$ & $180.18 \mathrm{~d}( \pm 0.092)$ \\
\hline
\end{tabular}

The different letter shows a significant difference in treatments at $5 \%$ probability level. $\mathrm{D}_{0}=$ Control, $\mathrm{D}_{1}=$ Drought at tillering stage, $D_{2}=$ Drought at grain filling, $B_{0}=$ (No-Biochar), $B_{1}=28 \mathrm{~g} \mathrm{~kg}^{-1}$ Biochar, $B_{2}=38 \mathrm{~g} \mathrm{~kg}^{-1}$ Biochar. Values in brackets are standard error of the mean $(n=4)$.

Soil organic carbon (SOC), dissolved organic nitrogen (DON), dissolved organic carbon (DOC), nitrogen mineralization (Nmin), and soil respiration (SR) were highest in soil under full irrigation with the highest biochar amendment, but were lowest under drought at grain filling stage without biochar addition, except SOC that was lowest under drought at the tillering stage at $28 \mathrm{~g} \mathrm{~kg}^{-1}$ biochar rate (Table 5). Generally, with or without biochar amendment, these parameters were greatest in the soil of fully irrigated crops, but were lowest under drought at the grain filling stage, except SR that showed no difference between both drought conditions at $28 \mathrm{~g} \mathrm{~kg}^{-1}$ biochar rate with the lower biochar rate. Except that SR did not differ between both drought conditions with the lower biochar rate.

Table 5. Effect of Biochar on SOC (Soil organic C), DOC (Dissolved organic C), DON (Dissolved organic N), N $N_{\text {MIN }}($ Nitrogen Mineralization), and SR (Soil Respiration) under water-limited stress condition.

\begin{tabular}{|c|c|c|c|c|c|}
\hline Treatments & $\operatorname{SOC}\left(\mathrm{g} \mathrm{kg}^{-1}\right)$ & DOC $\left(\mu g g^{-1}\right)$ & DON ( $\left.\mu g g^{-1}\right)$ & $\mathbf{N}_{\text {MIN }}$ & SR \\
\hline $\mathrm{D}_{0}$ & $1.34 \mathrm{~A}( \pm 0.0016)$ & $143.83 \mathrm{~A}( \pm 0.083)$ & $21.85 \mathrm{~A}( \pm 0.093)$ & $77.52 \mathrm{~A}( \pm 0.098)$ & $23.86 \mathrm{~A}( \pm 0.018)$ \\
\hline $\mathrm{D}_{1}$ & $1.30 \mathrm{~B}( \pm 0.0017)$ & 138.48 B $( \pm 0.078)$ & $19.81 \mathrm{~B}( \pm 0.079)$ & $65.14 \mathrm{~B}( \pm 0.076)$ & $22.38 \mathrm{~B}( \pm 0.220)$ \\
\hline $\mathrm{D}_{2}$ & $1.31 \mathrm{C}( \pm 0.0009)$ & $133.56 \mathrm{C}( \pm 0.127)$ & $15.81 \mathrm{C}( \pm 0.076)$ & $51.85 \mathrm{C}( \pm 0.094)$ & $21.24 \mathrm{C}( \pm 0.005)$ \\
\hline $\mathrm{B}_{0}$ & $0.96 C( \pm 0.0058)$ & $129.48 \mathrm{C}( \pm 2.299)$ & $16.46 \mathrm{C}( \pm 1.773)$ & $53.53 \mathrm{C}( \pm 6.027)$ & $20.85 C( \pm 0.585)$ \\
\hline $\mathrm{B}_{1}$ & $1.48 \mathrm{~B}( \pm 0.0177)$ & 135.17 B $( \pm 2.906)$ & $19.48 \mathrm{~B}( \pm 1.761)$ & 65.16 B $( \pm 8.667)$ & 22.42 B $( \pm 0.929)$ \\
\hline $\begin{array}{c}\mathrm{B}_{2} \\
\mathrm{D} \times \mathrm{B}\end{array}$ & $1.52 \mathrm{~A}( \pm 0.0122)$ & $151.21 \mathrm{~A}( \pm 3.749)$ & $21.53 \mathrm{~A}( \pm 1.782)$ & $75.82 \mathrm{~A}( \pm 8.072)$ & $23.85 \mathrm{~A}( \pm 0.821)$ \\
\hline $\mathrm{D}_{0} \mathrm{~B}_{0}$ & $0.97 \mathrm{~d}( \pm 0.0011)$ & 133.11 ef $( \pm 0.060)$ & $19.16 \mathrm{~d}( \pm 0.080)$ & 65.24 c $( \pm 0.121)$ & $22.14 \mathrm{~b}( \pm 0.012)$ \\
\hline $\mathrm{D}_{0} \mathrm{~B}_{1}$ & $1.52 \mathrm{ab}( \pm 0.0017)$ & $140.18 \mathrm{~d}( \pm 0.097)$ & $22.19 \mathrm{~b}( \pm 0.083)$ & $80.18 \mathrm{~b}( \pm 0.094)$ & $24.25 \mathrm{ab}( \pm 0.014)$ \\
\hline $\mathrm{D}_{0} \mathrm{~B}_{2}$ & $1.54 \mathrm{a}( \pm 0.0021)$ & 158.18 a $( \pm 0.093)$ & 24.23 a $( \pm 0.116)$ & 87.15 a $( \pm 0.078)$ & 25.18 a $( \pm 0.031)$ \\
\hline $\mathrm{D}_{1} \mathrm{~B}_{0}$ & $0.96 \mathrm{de}( \pm 0.0012)$ & $130.11 \mathrm{f}( \pm 0.066)$ & $17.11 \mathrm{f}( \pm 0.066)$ & 50.16 e $( \pm 0.105)$ & $21.34 \mathrm{~cd}( \pm 0.008)$ \\
\hline $\mathrm{D}_{1} \mathrm{~B}_{1}$ & $1.46 \mathrm{~cd}( \pm 0.0024)$ & 135.19 e $( \pm 0.102)$ & 20.11 c $( \pm 0.068)$ & $65.14 \mathrm{c}( \pm 0.073)$ & 21.77 c $( \pm 0.665)$ \\
\hline $\mathrm{D}_{1} \mathrm{~B}_{2}$ & $1.52 \mathrm{ab}( \pm 0.0023)$ & $150.11 \mathrm{~b}( \pm 0.066)$ & $22.18 \mathrm{~b}( \pm 0.105)$ & $80.12 \mathrm{~b}( \pm 0.062)$ & $24.03 \mathrm{~b}( \pm 0.003)$ \\
\hline $\mathrm{D}_{2} \mathrm{~B}_{0}$ & 0.95 e $( \pm 0.0008)$ & $125.22 \mathrm{~g}( \pm 0.124)$ & 13.12 h $( \pm 0.069)$ & $45.19 \mathrm{f}( \pm 0.105)$ & 20.13 e $( \pm 0.005)$ \\
\hline $\mathrm{D}_{2} \mathrm{~B}_{1}$ & 1.48 c $( \pm 0.0007)$ & $130.12 \mathrm{f}( \pm 0.069)$ & $16.16 \mathrm{~g}( \pm 0.081)$ & 50.16 e $( \pm 0.080)$ & $21.23 \mathrm{~d}( \pm 0.006)$ \\
\hline $\mathrm{D}_{2} \mathrm{~B}_{2}$ & $1.50 \mathrm{~b}( \pm 0.0011)$ & 145.33 c $( \pm 0.187)$ & 18.16 e $( \pm 0.084)$ & $60.20 \mathrm{~d}( \pm 0.102)$ & 22.35 c $( \pm 0.003)$ \\
\hline
\end{tabular}

The different letter shows a significant difference in treatments at $5 \%$ probability level. $\mathrm{D}_{0}=$ Control, $\mathrm{D}_{1}=$ Drought at tillering stage, $\mathrm{D}_{2}=$ Drought at grain filling, $\mathrm{B}_{0}=($ No-Biochar $), \mathrm{B}_{1}=28 \mathrm{~g} \mathrm{~kg}^{-1}$ Biochar, $\mathrm{B}_{2}=38 \mathrm{~g} \mathrm{~kg}^{-1}$ Biochar. Values in brackets are standard error of the mean $(n=4)$. 
Microbial biomass carbon (MBC), phosphorus (MBP), and nitrogen (MBN) were highest in the soil under full irrigation with the highest biochar amendment $\left(38 \mathrm{~g} \mathrm{~kg}^{-1}\right)$, but were lowest when drought was imposed at grain filling without biochar addition (Figures 1 and 2). Under the different soil moisture conditions (full irrigation or drought), these soil parameters were greatest with the highest biochar rate but were lowest in unamended soil. Generally, with or without biochar amendment, these parameters were greatest in the soil of fully irrigated crops but were lowest under drought at the grain filling stage.

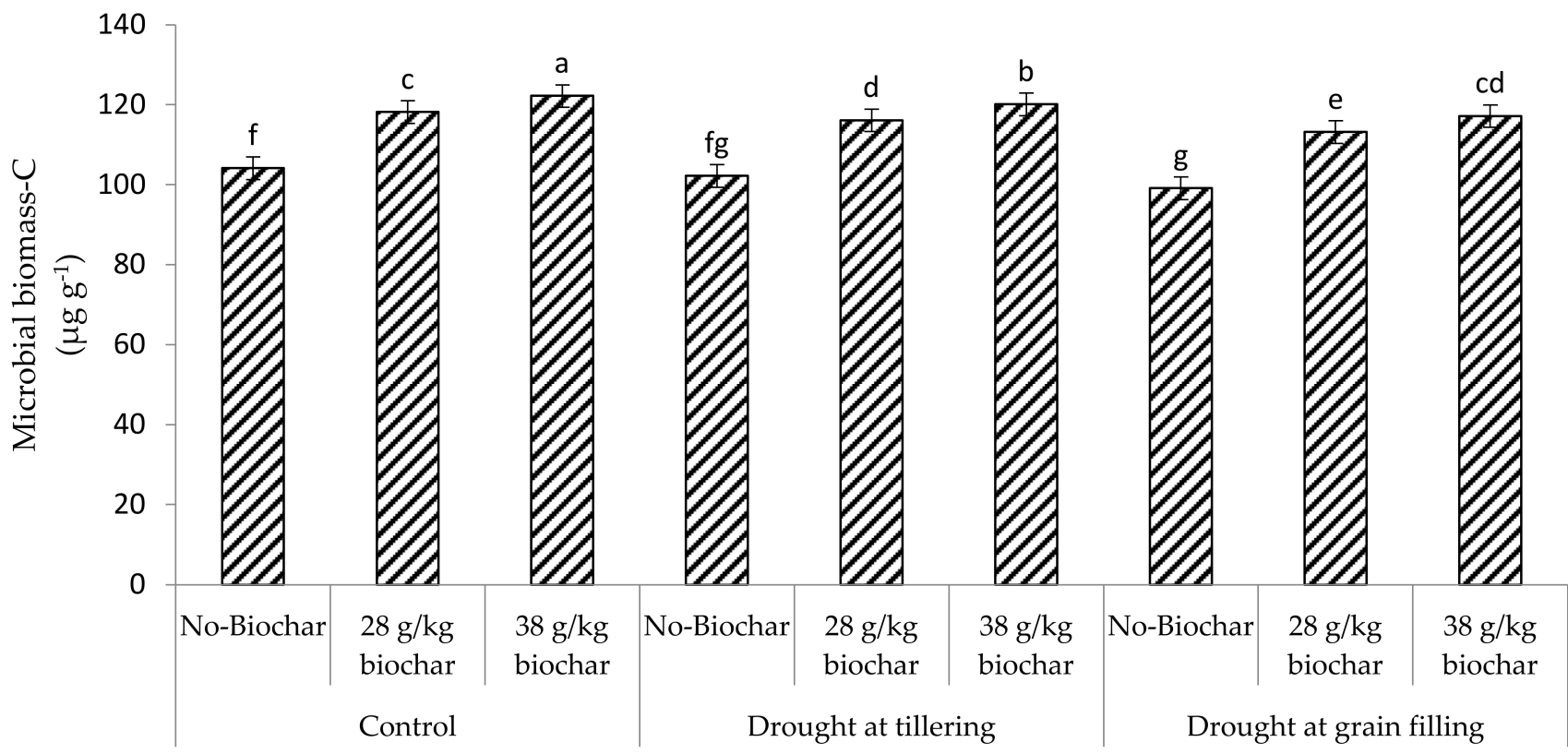

Figure 1. Effect of Biochar on Microbial biomass-C of soil under drought.

a Microbial biomass-N $\quad$ Microbial biomass-P

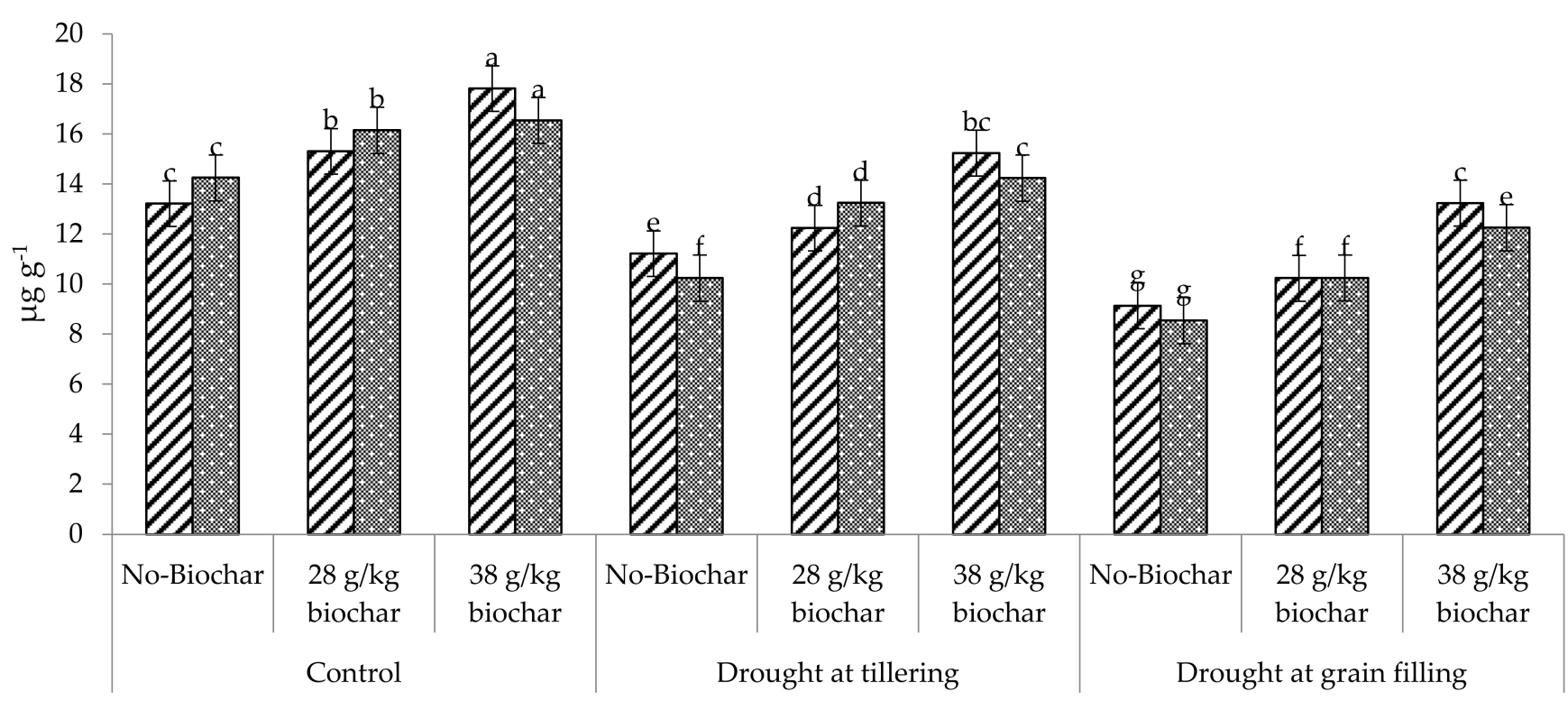

Figure 2. Effect of Biochar on Microbial biomass-N and Microbial biomass-P of soil under drought.

Data regarding different enzymatic activities (Table 6) in the soil of wheat crop shows that the highest soil dehydrogenase (DH), urease (UR), acid phosphatase (AcP), and $\beta$ glucosidase $(\beta G)$ were noticed when there is no drought followed by drought at grain filling 
and lowest was noticed when drought applied at grain filling stage. Biochar significantly affects the soil enzymatic activities as highest soil enzymatic activities were noticed when biochar applied with the rate of $38 \mathrm{~g} \mathrm{~kg}^{-1}$ followed by when applied biochar with the rate of $28 \mathrm{~g} \mathrm{~kg}^{-1}$ and lowest enzymatic activities were observed in a treatment having no biochar. A significant interaction was also noticed between biochar application and drought treatments.

Table 6. Effect of Biochar on Soil dehydrogenase (DH), urease (UR), acid phosphatase (AcP), and $\beta$-glucosidase ( $\beta \mathrm{G})$ activities in the soil of wheat crop under water-limited stress condition.

\begin{tabular}{|c|c|c|c|c|}
\hline Treatments & 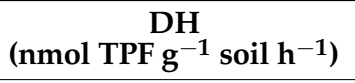 & $\begin{array}{c}\mathrm{UR} \\
\left(\mu \mathrm{mol} \mathrm{NH}{ }_{3}-\mathrm{N} \mathrm{g}^{-1} \mathrm{~h}^{-1}\right)\end{array}$ & $\begin{array}{c}\text { AcP } \\
\left(\mu \mathrm{mol} p \text {-Nitrophenol } \mathrm{g}^{-1} \mathrm{~h}^{-1}\right)\end{array}$ & $\begin{array}{c}\beta G \\
\left(\mu \mathrm{mol} p \text {-Nitrophenol } \mathrm{g}^{-1} \mathrm{~h}^{-1}\right)\end{array}$ \\
\hline $\mathrm{D}_{0}$ & $70 \mathrm{~A}( \pm 0.816)$ & $15.34 \mathrm{~A}( \pm 0.278)$ & $26.33 \mathrm{~A}( \pm 0.247)$ & $6.65 \mathrm{~A}( \pm 0.064)$ \\
\hline$D_{1}$ & $35 \mathrm{C}( \pm 1.080)$ & $09.32 \mathrm{C}( \pm 0.123)$ & $17.45 \mathrm{C}( \pm 0.170)$ & $2.45 \mathrm{C}( \pm 0.090)$ \\
\hline $\mathrm{D}_{2}$ & $67 \mathrm{~B}( \pm 0.912)$ & $12.34 \mathrm{~B}( \pm 0.108)$ & 21.67 B $( \pm 0.141)$ & $4.53 \mathrm{~B}( \pm 0.067)$ \\
\hline $\mathrm{B}_{0}$ & $67 C( \pm 1.354)$ & $16.12 \mathrm{C}( \pm 0.021)$ & $24.54 \mathrm{C}( \pm 0.272)$ & $5.25 \mathrm{C}( \pm 0.322)$ \\
\hline $\mathrm{B}_{1}$ & $76 \mathrm{~B}( \pm 1.020)$ & 17.33 B $( \pm 0.086)$ & $28.34 \mathrm{~B}( \pm 0.236)$ & $6.43 \mathrm{~B}( \pm 0.237)$ \\
\hline $\mathrm{B}_{2}$ & $82 \mathrm{~A}( \pm 0.790)$ & 21.35 A $( \pm 0.027)$ & $32.23 \mathrm{~A}( \pm 0.453)$ & $6.90 \mathrm{~A}( \pm 0.279)$ \\
\hline $\begin{array}{l}\mathrm{D} \times \mathrm{B} \\
\mathrm{D}_{0} \mathrm{~B}_{0}\end{array}$ & $65 c( \pm 0.912)$ & $13.23 \mathrm{c}( \pm 0.099)$ & $23.43 c( \pm 0.113)$ & $6.12 c( \pm 0.094)$ \\
\hline $\mathrm{D}_{0} \mathrm{~B}_{1}$ & $70 \mathrm{~b}( \pm 1.080)$ & $16.34 \mathrm{~b}( \pm 0.128)$ & $27.76 \mathrm{~b}( \pm 0.540)$ & $6.86 \mathrm{~b}( \pm 0.199)$ \\
\hline $\mathrm{D}_{0} \mathrm{~B}_{2}$ & 75 a $( \pm 0.912)$ & 18.31a $( \pm 0.257)$ & 31.33 a $( \pm 0.235)$ & 7.02 a $( \pm 0.366)$ \\
\hline $\mathrm{D}_{1} \mathrm{~B}_{0}$ & $31 \mathrm{~h}( \pm 1.354)$ & $04.43 \mathrm{~g}( \pm 0.257)$ & $14.56 \mathrm{~g}( \pm 0.536)$ & $2.04 \mathrm{~h}( \pm 0.138)$ \\
\hline $\mathrm{D}_{1} \mathrm{~B}_{1}$ & 35 gh $( \pm 0.707)$ & $06.12 \mathrm{f}( \pm 0.069)$ & $16.23 \mathrm{fg}( \pm 0.468)$ & $3.12 \mathrm{gh}( \pm 0.042)$ \\
\hline $\mathrm{D}_{1} \mathrm{~B}_{2}$ & $39 \mathrm{~g}( \pm 0.790)$ & 08.34 ef $( \pm 0.057)$ & $17.34 \mathrm{f}( \pm 0.703)$ & $3.65 \mathrm{f}( \pm 0.165)$ \\
\hline $\mathrm{D}_{2} \mathrm{~B}_{0}$ & $61 \mathrm{f}( \pm 0.540)$ & 09.45 e $( \pm 0.101)$ & $17.43 \mathrm{f}( \pm 0.592)$ & 3.89 e $( \pm 0.067)$ \\
\hline $\mathrm{D}_{2} \mathrm{~B}_{1}$ & 65 e $( \pm 0.472)$ & $11.62 \mathrm{~d}( \pm 0.099)$ & 19.21 e $( \pm 0.459)$ & $4.12 \mathrm{de}( \pm 0.131)$ \\
\hline $\mathrm{D}_{2} \mathrm{~B}_{2}$ & $70 \mathrm{~d}( \pm 0.965)$ & 12.77 c $( \pm 0.170)$ & $21.65 \mathrm{~d}( \pm 0.788)$ & $4.67 \mathrm{~d}( \pm 0.118)$ \\
\hline
\end{tabular}

The different letter shows a significant difference in treatments at $5 \%$ probability level. $\mathrm{D}_{0}=$ Control, $\mathrm{D}_{1}=$ Drought at tillering stage, $\mathrm{D}_{2}=$ Drought at grain filling, $\mathrm{B}_{0}=($ No-Biochar $), \mathrm{B}_{1}=28 \mathrm{~g} \mathrm{~kg}^{-1}$ Biochar, $\mathrm{B}_{2}=38 \mathrm{~g} \mathrm{~kg}^{-1}$ Biochar. Values in brackets are standard error of the mean $(n=4)$.

\section{Discussion}

The study shows that drought stress at both tillering and grain filling stages significantly lowered soil $\mathrm{pH}$, whereas higher $\mathrm{pH}$ was maintained in constantly irrigated soils. Similar results were reported by Siebielec et al. [11]. Zhang et al. [27] also found that soil $\mathrm{pH}$ significantly decreased under water stress conditions, which was attributed to poor solubilization of phosphate compounds.

Growth and yield-related parameters of the wheat crop are interlinked with each other. The higher 1000-grain weight leads towards the higher grain yield per plant [2]. Zaheer et al. [4] reported that drought stress at any growth stage severely damages wheat growth and yield as water is essential for plant growth and development. Under drought stress, there is less availability of the soil nutrients and less production of the photosynthetic material in the plant so there is less plant growth. Biochar is an effective soil amendment as it improves soil properties and maintains long-term soil productivity [28]. Biochar application as a soil amendment has a positive effect on plant growth and yield [29]. Biochar can enhance the soil carbon and organic matter contents in the soil, and it can also improve the soil microbial activities that enhance the soil nutrients availability [30].

Under low soil water conditions, such as the drought condition, the accumulation of organic matter may contribute to lower soil pH through the release of hydrogen ions [31]. Also, the increased polarity of accumulated cations due to soil drying may lower the soil $\mathrm{pH}$ [32]. Song et al. [33] also showed that a decrease in soil pH during water stress could indicate that moisture enhances the correlativity between phosphatase activity in the soil.

However, drought-induced decreases in soil $\mathrm{pH}$ were ameliorated with the addition of biochar. Previous studies have also reported that biochar increased soil pH [34-38]. An increase in soil $\mathrm{pH}$ due to biochar application has been attributed to the release of inherent alkaline substances [39] and possibly high calcium carbonate content and the calcium carbonate equivalent (CCE) [40]. According to Yuan et al. [36], the alkalinity of biochar is 
an important factor that affects their liming potential. In our study, the effect of biochar on soil $\mathrm{pH}$ increased with increasing biochar rate across the different soil water conditions.

Exposure to drought significantly lowered nutrients (total $\mathrm{N}$, mineral $\mathrm{N}$, bray $\mathrm{P}$, and exchangeable K) concentrations in the soil, compared with constantly irrigated soils. Under drought conditions, lower soil water content limits microbial activity, resulting in reduced organic matter decomposition. Marschner [41] found that soil respiration and phosphorus pools were lower in soil with low $(-1.700 \mathrm{MPa})$ water content in comparison with soil with optimum $(-0.078 \mathrm{MPa})$ water content. Lower microbial activity and $\mathrm{P}$ availability in soil exposed to drought compared with constantly irrigated soils [4]. Higher soil nutrient content in constantly irrigated soils could suggest the effects of soil microbes in decomposing soil organic matter, resulting in nutrient mineralization [2].

Biochar amendment increased soil nutrients (total $\mathrm{N}$, mineral $\mathrm{N}$, bray $\mathrm{P}$, and exchangeable $\mathrm{K}$ ) concentrations in both constantly irrigated and drought exposed soils when compared with unamended soils. Similar results were observed in Karimi et al. [42]. Ahmed et al. [30] also corroborated that biochar application increased nitrogen retention and soil organic matter in the soil, resulting in improved water use efficiency. The biocharinduced increase in soil nutrient availability in the soil under regular watering and droughtimposed treatments could be due both to the release of available nutrients from the biochar itself or enhanced decomposition of native soil organic matter, referred to as "soil priming" [43]. Shen et al. [44] also showed that biochar application to the soil enhanced nutrient availability and improved soil fertility. The biochar pyrolysis temperature and source have been attributed to be significant factors in determining the concentration of nutrients released into amended soil $[28,29,45]$.

Drought stress condition decreases large macroaggregates of SOC pool which in turn affects soil stability. Carbon metabolism is highly sensitive to soil moisture [46]. DOC, SOC, and DON are related to the availability of soil moisture and they are all reduced under water stress conditions. Conversely, Ritson et al. [47] found that water stress caused an increase in DOC production from peatland. Hailegnaw et al. [48] reported that most of the biochar is carbon so its addition to soil also enhances the SOC and DOC. Biochar also enhances the organic matter contents in the soil and microbial activities, thereby improving DON [49].

Bond-Lamberty et al. [50] reported that nitrogen mineralization is also affected by the availability of soil moisture. Drought condition increases nitrogen loss due to the increase in asynchronicity between the nitrogen released by the mineralization and its uptake by the plant. Any soil amendments that can enhance organic matter in the soil could mitigate the loss of nitrogen. The application of biochar also enhances soil organic matter and slows down the loss of nitrogen, so its application enhances the nitrogen mineralization in the soil [48].

Soil respiration is very important for sustainable plant growth, microorganisms, and soil fauna. It indicates the rate of soil organic matter decomposition and microbial activities [49]. Findings from this study showed that SR decreased by $6.02 \%$ and $10.98 \%$ under water stress conditions at the tillering and grain filling stage, respectively, but when biochar was applied and then subjected to water stress at the same stages, significantly higher SR was reported. This is in line with the work of Skopp et al. [51] who reported that the limited soil moisture decreased the microbial activities in the soil and more of these microbes and plant roots invest energy to produce protective molecules that decrease the soil respiration. Smith et al. [52] noticed that biochar increases soil carbon, organic matter, and microbial activities in the soil, which also enhances the soil respiration.

Soil microbial biomass plays a vital role in the decomposition of organic matter. Higher microbial biomass enhances soil fertility and increases the availability of soil nutrients. It also acts as the linkage of the source and sinks between soil nutrients [41]. Sanaullah et al. [53] reported that drought stress condition creates osmotic stress and causes microbial death that creates microbial biomass reduction. More of this organic matter decomposition also decreases with drought stress. Hailegnaw et al., [48], Smith et al. [52], 
Al-Wabel et al. [49], Martinsen et al. [54], Cornelissen et al. [55], and many other researchers reported that biochar application enhanced the microbial activities, organic matter, and nutrient content in the soil thereby improving the microbial biomass of the soil, which is helpful in the improvement of soil fertility and plant growth.

Findings show that drought stress causes a considerable decrease in the soil enzymatic activities. Li and Sarah [56] also reported that enzymatic activities in soil decreased due to the climatic transect under limited water stress. Unavailability of the soil water decreases the soil microbial activities involved in the recycling of various soil nutrients. Drought stress at different growth stages of wheat has a different effect on the soil enzymatic activities as there is a different level of soil nutrients status and different requirements for plant growth. Mayor et al. [57] reported that limited Nitrogen availability under drought conditions leads to a decrease in enzymatic activities. Our findings are in agreement with previous researchers as phosphate activities, urease, $\beta$-glucosidase, and dehydrogenase correlated with the availability of soil water [58,59], however, activities of urease under limited availability of water are not always affected [60]. Biochar application also increases the organic carbon that can be very helpful to enhance the soil microflora. Microbial activities in the soil also enhance the organic matter and nutrients status of the soil. Many researchers [61-68] also reported the increase of the organic matter in the soil due to biochar also enhances the soil enzymatic activities and impact on crops [69]. Zhao et al. [70] reported that biochar application as soil amendments increase the soil organic matter contents that are helpful in the soil health and quality.

\section{Conclusions}

Biochar application was found to have a significant effect on the soil's biochemical properties. Most of the organic matter significantly increased with the application of biochar. Biochar application under water stress conditions can improve the plant growth and chemical properties of soil. Biochar application with the rate of $38 \mathrm{~g} \mathrm{~kg}^{-1}$ of soil under water stress conditions is more beneficial in the comparison with $28 \mathrm{~g} \mathrm{~kg}^{-1}$ rate. Soil carbon contents and mineral nutrients also increased with the application of biochar, so it is recommended to farmers and other crop plant researchers to use biochar especially under drought conditions to improve plant growth and also as an alternative means of improving nutrient availability status for plant growth.

Author Contributions: Conceptualization, M.S.Z., H.H.A., and W.S.; methodology, M.S.Z., H.H.A., and R.I., analysis and validation, A.E.S., W.S.; R.I.; and M.H.-u.-R., writing-original draft preparation, M.S.Z., M.I. and J.I., writing-review and editing, M.H.-u.-R., and W.S., and A.E.S.; funding acquisition, W.S., and H.H.A.; supervision, M.H.-u.-R. All authors have read and agreed to the published version of the manuscript.

Funding: This Research was funded by the Researchers Supporting Project number (RSP-2021/390), King Saud University, Riyadh, Saudi Arabia.

Institutional Review Board Statement: Not applicable.

Informed Consent Statement: Not applicable.

Data Availability Statement: Data will be available on reasonable request from first and corresponding authors.

Acknowledgments: The technical support for this study was provided by the Regional Agriculture Research Institute in Bahawalpur. Ayub Agricultural Research Institute in Faisalabad and Khwaja Fareed University of Engineering and Information Technology, Rahim Yar Khan, Pakistan is also greatly acknowledged. This Research was funded by the Researchers Supporting Project number (RSP-2021/390), King Saud University, Riyadh, Saudi Arabia.

Conflicts of Interest: The authors declare no conflict of interest. 


\section{References}

1. Dinu, M.; Whittaker, A.; Pagliai, G.; Benedettelli, S.; Sofi, F. Ancient wheat species and human health: Biochemical and clinical implications. J. Nutr. Biochem. 2018, 52, 1-9. [CrossRef]

2. Zaheer, M.S.; Raza, M.A.S.; Saleem, M.F.; Khan, I.H.; Ahmad, S.; Iqbal, R.; Manevski, K. Investigating the effect of Azospirillum brasilense and Rhizobium pisi on agronomic traits of wheat (Triticum aestivum L.). Arch. Agron. Soil Sci. 2019, 65, 1554-1564. [CrossRef]

3. Zhang, J.; Zhang, S.; Cheng, M.; Jiang, H.; Zhang, X.; Peng, C.; Lu, X.; Zhang, M.; Jin, J. Effect of drought on agronomic traits of rice and wheat: A meta-analysis. Int. J. Environ. Res. Public Health 2018, 15, 839. [CrossRef] [PubMed]

4. Zaheer, M.S.; Raza, M.A.S.; Saleem, M.F.; Erinle, K.O.; Iqbal, R.; Ahmad, S. Effect of rhizobacteria and cytokinins application on wheat growth and yield under normal vs drought conditions. Commun. Soil Sci. Plant Anal. 2019, 50, 2521-2533. [CrossRef]

5. Onyekachi, O.G.; Boniface, O.O.; Gemlack, N.F.; Nicholas, N. The Effect of Climate Change on Abiotic Plant Stress: A Review. In Abiotic and Biotic Stress in Plants; IntechOpen: London, UK, 2018. [CrossRef]

6. Garcia-Vila, M.; Morillo-Velarde, R.; Fereres, E. Modeling Sugar Beet Responses to Irrigation with AquaCrop for Optimizing Water Allocation. Water 2019, 11, 1918. [CrossRef]

7. Scotti, R.; Ascoli, R.D.; Rao, M.A.; Marzaioli, R.; Rutigliano, F.A.; Gianfreda, L. Impact of an intensive management on soil biochemical and biological properties in an agricultural soil of Southern Italy. Geophys. Res. Abstr. 2009, 11, 13268.

8. Biswas, S.; Hazra, G.C.; Purakayastha, T.; Saha, N.; Mitran, T.; Roy, S.S.; Basak, N.; Mandal, B. Establishment of critical limits of indicators and indices of soil quality in rice-rice cropping systems under dierent soilorders. Geoderma 2017, 292, 34-48. [CrossRef]

9. Wang, X.; Gong, Z. Assessment and analysis of soil quality changes after eleven years of reclamation in subtropical China. Geoderma 1998, 81, 339-355. [CrossRef]

10. Zheng, H.; Wang, X.; Luo, X.; Wang, Z.; Xing, B. Biochar-induced negative carbon mineralization priming effects in a coastal wetland soil: Roles of soil aggregation and microbial modulation. Sci. Total Environ. 2018, 610, 951-960. [CrossRef]

11. Siebielec, S.; Siebielec, G.; Klimkowicz-Pawlas, A.; Gałązka, A.; Grządziel, J.; Stuczyński, T. Impact of Water Stress on Microbial Community and Activity in Sandy and Loamy Soils. Agronomy 2020, 10, 1429. [CrossRef]

12. Zhang, M.; Riaz, M.; Zhang, L.; El-Desouki, Z.; Jiang, C. Biochar Induces Changes to Basic Soil Properties and Bacterial Communities of Different Soils to Varying Degrees at $25 \mathrm{~mm}$ Rainfall: More Effective on Acidic Soils. Front. Microbiol. 2019, 10, 1321. [CrossRef]

13. Ali, S.; Rizwan, M.; Qayyum, M.F.; Ok, Y.S.; Ibrahim, M.; Riaz, M.; Arif, M.S.; Hafeez, F.; Al-Wabel, M.I.; Shahzad, A.N. Biochar soil amendment on alleviation of drought and salt stress in plants: A critical review. Environ. Sci. Pollut. Res. 2017, 24, 12700-12712. [CrossRef] [PubMed]

14. Baiamonte, G.; De Pasquale, C.; Marsala, V.; Cimò, G.; Alonzo, G.; Crescimanno, G.; Conte, P. Structure alteration of a sandy-clay soil by biochar amendments. J. Soils Sedim. 2015, 15, 816-824. [CrossRef]

15. Odugbenro, G.O.; Liu, Z.; Sun, Y. Soil aggregate size distribution and total organic carbon in intra-aggregate fractions as affected by addition biochar and organic amendments. Pol. J. Soil Sci. 2020, 53, 41-54. [CrossRef]

16. Conte, P.; Hanke, U.M.; Marsala, V.; Cimo, G.; Alonzo, G.; Glaser, B. Mechanisms of water interaction with pore systems of hydrochar and pyrochar from poplar forestry waste. J. Agric. Food Chem. 2014, 62, 4917-4923. [CrossRef]

17. Brantley, K.E.; Brye, K.R.; Savin, M.C.; Longer, D.E. Biochar source and application rate effects on soil water retention determined using wetting curves. Open J. Soil Sci. 2015, 5, 1-10. [CrossRef]

18. Rehman, M.Z.U.; Rizwan, M.; Ali, S.; Fatima, N.; Yousaf, B.; Naeem, A.; Ok, Y.S. Contrasting effects of biochar, compost and farm manure on alleviation of nickel toxicity in maize (Zea mays L.) in relation to plant growth, photosynthesis and metal uptake. Ecotoxicol. Environ. Saf. 2016, 133, 218-225. [CrossRef]

19. Sparks, D.L.; Page, A.L.; Helmke, P.A.; Loeppert, R.H.; Soltanpour, P.N.; Tabatabai, M.A.; Johnston, C.T.; Sumner, M.E. Methods of Soil Analysis, Part 3, Chemical Methods; SSSA: Madison, ME, USA, 1996.

20. Dinesh, R.; Anandaraj, M.; Kumar, A.; Srinivasan, V.; Bini, Y.K.; Subila, K.P.; Aravind, R.; Hamza, S. Effects of Plant GrowthPromoting Rhizobacteria and NPK Fertilizers on Biochemical and Microbial Properties of Soils Under Ginger (Zingiber officinale) Cultivation. Agric. Res. 2013, 2, 346-353. [CrossRef]

21. Mulvaney, R.L. Nitrogen-inorganic forms. In Methods of Soil Analysis, Part 3, Chemical Methods; Sparks, D.L., Page, A.L., Helmke, P.A., Eds.; SSSA Book Series, Number 5 Madison; SSSA: Madison, ME, USA, 1996; pp. 1123-1184.

22. Smolander, A.; Kitunen, V. Soil microbial activities and characteristics of dissolved organic $\mathrm{C}$ and $\mathrm{N}$ in relation to tree species. Soil Biol. Biochem. 2002, 34, 651-660. [CrossRef]

23. Vance, E.D.; Brookes, P.C.; Jenkinson, D.S. An extraction method for measuring soil microbial biomass C. Soil Biol. Biochem. 1987, 19, 703-707. [CrossRef]

24. Heinze, S.; Rauber, R.; Joergensen, R.G. Influence of mouldboard plough and rotary harrow tillage on microbial biomass and nutrient stocks in two long-term experiments on loess derived Luvisols. Appl. Soil Ecol. 2010, 46, 405-412. [CrossRef]

25. Tabatabai, M.A. Soil enzymes. In Methods of Soil Analysis, Part 2, Microbiological and Biochemical Properties; Weaver, R.W., Angle, S., Bottomley, P., Bezdicek, D., Smith, S., Tabatabai, A., Wollum, A., Eds.; SSSA: Madison, ME, USA, 1994; pp. $775-833$.

26. Steel, R.G.D.; Torrie, J.H.; Dickey, D.A. Principles and Procedures of Statistics, a Biometrical Approach, 3rd ed.; McGraw-Hill: New York, NY, USA, 1997; pp. 352-358. 
27. Zhang, H.; Shi, L.; Lu, H.; Shao, Y.; Liu, S.; Fu, S. Drought promotes soil phosphorus transformation and reduces phosphorus bioavailability in a temperate forest. Sci. Total Environ. 2020, 732, 139295. [CrossRef] [PubMed]

28. Wang, Y.; Lin, Y.; Chiu, P.C.; Imhoff, P.T.; Guo, M. Phosphorus release behaviors of poultry litter biochar as a soil amendment. Sci. Total Environ. 2015, 512, 454-463. [CrossRef] [PubMed]

29. Naeem, M.A.; Khalid, M.; Aon, M.; Abbas, G.; Tahir, M.; Amjad, M.; Murtaza, B.; Yang, A.; Akhtar, S.S. Effect of wheat and rice straw biochar produced at different temperatures on maize growth and nutrient dynamics of a calcareous soil. Arch. Agron. Soil Sci. 2017, 63, 2048-2061. [CrossRef]

30. Ahmed, R.; Li, Y.; Mao, L.; Xu, C.; Lin, W.; Ahmed, S.; Ahmed, W. Biochar Effects on Mineral Nitrogen Leaching, Moisture Content, and Evapotranspiration after 15N Urea Fertilization for Vegetable Crop. Agronomy 2019, 9, 331. [CrossRef]

31. Ritchie, G.S.P.; Dolling, P.J. The role of organic matter in soil acidification. Aust. J. Soil Res. 1985, 23, 569-576. [CrossRef]

32. Bartlett, R.; James, B. Studying Dried, Stored Soil Samples-Some Pitfalls. Soil Sci. Soc. Am. J. 1980, 44, 721-724. [CrossRef]

33. Song, F.; Han, X.; Zhu, X.; Herbert, S.J. Response to water stress of soil enzymes and root exudates from drought and non-drought tolerant corn hybrids at different growth stages. Can. J. Soil Sci. 2012, 92, 501-507. [CrossRef]

34. Van Zwieten, L.; Kimber, S.; Morris, S.; Chan, K.; Downie, A.; Rust, J.; Joseph, S.; Cowie, A. Effects of biochar from slow pyrolysis of papermill waste on agronomic performance and soil fertility. Plant Soil 2010, 327, 235-246. [CrossRef]

35. Fellet, G.; Marchiol, L.; Delle Vedove, G.; Peressotti, A. Application of biochar on mine tailings: Effects and perspectives for land reclamation. Chemosphere 2011, 83, 1262-1267. [CrossRef] [PubMed]

36. Yuan, J.; Xu, R.; Wang, N.; Li, J. Amendment of acid soils with crop residues and biochars. Pedosphere 2011, 21, 302-308. [CrossRef]

37. Yao, L.; Yu, X.; Huang, L.; Zhang, X.; Wang, D.; Zhao, X.; Li, Y.; He, Z.; Kang, L.; Li, X.; et al. Responses of Phaseolus calcaltus to lime and biochar application in an acid soil. PeerJ 2019, 7, e6346. [CrossRef] [PubMed]

38. Shetty, R.; Prakash, N.B. Effect of different biochars on acid soil and growth parameters of rice plants under aluminium toxicity. Sci Rep. 2020, 10, 12249. [CrossRef]

39. Liu, X.H.; Zhang, X.C. Effect of biochar on $\mathrm{pH}$ of alkaline soils in the loess plateau: Results from incubation experiments. Int. J. Agric. Biol. 2012, 14, 745-750.

40. Chintala, R.; Mollinedo, J.; Schumacher, T.E.; Malo, D.D.; Julson, J.L. Effect of biochar on chemical properties of acidic soil. Arch Agron. Soil Sci. 2014, 60, 393-404. [CrossRef]

41. Marschner, P.; Hatam, Z.; Cavagnaro, T. Soil respiration, microbial biomass and nutrient availability after the second amendment are influenced by legacy effects of prior residue addition. Soil Biol. Biochem. 2015, 88, 169-177. [CrossRef]

42. Karimi, A.; Moezzi, A.; Chorom, M.; Enayatizamir, N. Application of Biochar Changed the Status of Nutrients and Biological Activity in a Calcareous Soil. J. Soil Sci. Plant. Nutr. 2020, 20, 450-459. [CrossRef]

43. Fang, Y.; Singh, B.; Singh, B.P. Effect of temperature on biochar priming effects and its stability in soils. Soil Biol. Biochem. 2015, 80, 136-145. [CrossRef]

44. Shen, Q.; Hedley, M.; Arbestain, M.C.; Kirschbaum, M.U.F. Can biochar increase the bioavailability of phosphorus? J. Soil Sci. Plant. Nutr. 2016, 16, 268-286. [CrossRef]

45. Song, D.; Tang, J.; Xi, X.; Zhang, S.; Liang, G.; Zhou, W.; Wang, X. Responses of soil nutrients and microbial activities to additions of maize straw biochar and chemical fertilization in a calcareous soil. Eur. J. Soil Biol. 2018, 84, 1-10. [CrossRef]

46. Su, X.; Su, X.; Yang, S.; Zhou, G.; Ni, M.; Wang, C.; Qin, H.; Zhou, X.; Deng, J. Drought changed soil organic carbon composition and bacterial carbon metabolizing patterns in a subtropical evergreen forest. Sci. Total Environ. 2020, 736, 139568. [CrossRef] [PubMed]

47. Ritson, J.P.; Brazier, R.E.; Graham, N.J.D.; Freeman, C.; Templeton, M.R.; Clark, J.M. The effect of drought on dissolved organic carbon DOC release from peatland soil and vegetation sources. Biogeoscience 2017, 14, 2891-2902. [CrossRef]

48. Hailegnaw, N.S.; Mercl, F.; Pračke, K.; Száková, L.; Tlustoš, P. Mutual relationships of biochar and soil pH, CEC, and exchangeable base cations in a model laboratory experiment. J. Soils Sediments 2019, 19, 2405-2416. [CrossRef]

49. Al-Wabel, M.I.; Hussain, Q.; Usman, A.R.A.; Ahmad, M.; Abduljabbar, A.; Sallam, A.S.; Ok, Y.S. Impact of biochar properties on soil conditions and agricultural sustainability: A review. Land Degrad. Dev. 2018, 29, 124-2161. [CrossRef]

50. Bond-Lamberty, B.; Bolton, H.; Fansler, S.; Heredia-Langner, A.; Liu, C.; McCue, L.A.; Smith, J.; Bailey, V. Soil Respiration and Bacterial Structure and Function after 17 Years of a Reciprocal Soil Transplant Experiment. PLoS ONE 2016, 11, e0150599. [CrossRef]

51. Skopp, J.; Jawson, M.D.; Doran, J.W. Steady-State Aerobic Microbial Activity as a Function of Soil Water Content. Soil Sci. Soc. Am. J. 1990, 54, 1619-1625. [CrossRef]

52. Smith, J.L.; Collins, H.P.; Bailey, V.L. The effect of young biochar on soil respiration. Soil Biol. Biochem. 2010, 42, 2345-2347. [CrossRef]

53. Sanaullah, M.; Blagodatskaya, E.; Chabbi, A.; Rumpel, C.; Kuzyakov, Y. Drought effects on microbial biomass and enzyme activities in the rhizosphere of grasses depend on plant community composition. Appl. Soil Ecol. 2011, 48, 38-44. [CrossRef]

54. Martinsen, V.; Alling, V.; Nurida, N.L.; Mulder, J.; Hale, S.E.; Ritz, C.; Rutherford, D.W.; Heikens, A.; Breedveld, G.D.; Cornelissen, G. pH effects of the addition of three biochars to acidic Indonesian mineral soils. Soil Sci. Plant. Nutr. 2015, 61, 821-834. [CrossRef]

55. Cornelissen, G.; Jubaedah, G.; Nurida, N.L.; Hale, S.E.; Martinsen, V.; Silvani, L.; Mulder, J. Fading positive effect of biochar on crop yield and soil acidity during five growth seasons in an Indonesian Ultisol. Sci. Total Environ. 2018, 634, 561-568. [CrossRef] 
56. Li, X.Z.; Sarah, P. Enzyme activities along a climatic transect in the Judean Desert. Catena 2003, 53, 349-363. [CrossRef]

57. Mayor, X.; Belmonte, R.; Rodrigo, A.; Rodà, F.; Pinõl, J. Crecimiento diametral de la encina (Quercus ilex L.) en unanõ de abundante precipitación estival: Efecto de la irrigación previa y de la fertilización. Orsis 1994, 9, 13-23.

58. Kramer, S.; Green, D.M. Acid and alkaline phosphatase dynamics and their relationship to soil microclimate in a semiarid woodland. Soil Biol. Biochem. 2000, 32, 179-188. [CrossRef]

59. Sardans, J.; Penuelas, J. Drought decreases soil enzyme activity in a Mediterranean Quercus ilex L. forest. Soil Biol. Biochem. 2005, 37, 455-461. [CrossRef]

60. Sall, C.N.; Chotte, J.L. Phosphatase and urease activities in a tropical sandy soil as affected by soil water-holding capacity and assay conditions. Commun. Soil Sci. Plant Anal. 2002, 33, 3745-3755. [CrossRef]

61. Zaman, M.; Di, H.J.; Cameron, K.C. A field study of gross rates of N mineralization and nitrification and their relationships to microbial biomass and enzyme activities in soils treated with dairy effluent and ammonium fertilizer. Soil Use Manag. 1999, 15, 188-194. [CrossRef]

62. Dodor, D.E.; Tabatabai, M.A. Effect of cropping systems on phosphatases in soils. J. Plant Nutr. Soil Sci. 2003, 166, 7-13. [CrossRef]

63. Ghafoor, I.; Habib-ur-Rahman, M.; Ali, M.; Afzal, M.; Ahmed, W.; Gaiser, T.; Ghaffar, A. Slow-release nitrogen fertilizers enhance growth, yield, NUE in wheat crop and reduce nitrogen losses under an arid environment. Environ. Sci. Pollut. Res. 2021, 28, 43528-43543. [CrossRef] [PubMed]

64. Rahman, M.H.; Ahmad, I.; Wang, D.; Fahad, S.; Afzal, M.; Ghaffar, A.; Saddique, Q.; Khan, M.A.; Saud, S.; Hassan, S.; et al. Influence of semi-arid environment on radiation use efficiency and other growth attributes of lentil crop. Environ. Sci. Pollut. Res. 2021, 28, 13697-13711. [CrossRef]

65. Rahman, M.H.; Ahmad, A.; Wang, X.; Wajid, A.; Nasim, W.; Hussain, M.; Ahmad, B.; Ahmad, I.; Ali, Z.; Ishaque, W.; et al. Multi-model projections of future climate and climate change impacts uncertainty assessment for cotton production in Pakistan. Agric. For. Meteorol. 2018, 253, 94-113. [CrossRef]

66. Khan, Z.; Rahman, M.; Haider, G.; Amir, R.; Ikram, R.; Ahmad, S.; Schofield, H.; Riaz, B.; Iqbal, R.; Fahad, S.; et al. Chemical and Biological Enhancement Effects of Biochar on Wheat Growth and Yield under Arid Field Conditions. Sustainability 2021, 13, 5890. [CrossRef]

67. Younis, U.; Rahi, A.A.; Danish, S.; Younis, U.; Ali, M.A.; Ahmed, N.; Datta, R.; Fahad, S.; Holatko, J.; Hammerschmiedt, T.; et al. Fourier Transform Infrared Spectroscopy vibrational bands study of Spinacia oleracea and Trigonella corniculata under biochar amendment in naturally contaminated soil. PLOS ONE 2021, 16, e0253390.

68. Haider, I.; Raza, M.A.S.; Iqbal, R.; Aslam, M.U.; Habib-ur-Rahman, M.; Raja, S.; Khan, M.T.; Aslam, M.M.; Waqas, M.; Ahmad, S. Potential effects of biochar application on mitigating the drought stress implications on wheat (Triticum aestivum L.) under various growth stages. J. Saudi Chem. Soc. 2020, 24, 974-981. [CrossRef]

69. Wang, H.; Ren, T.; Feng, Y.; Liu, K.; Feng, H.; Liu, G.; Shi, H. Effects of the Application of Biochar in Four Typical Agricultural Soils in China. Agronomy 2020, 10, 1649. [CrossRef]

70. Zhao, X.; Cheng, H.T.; Lu, G.H.; Jia, Q.Y. Research progress on soil microbial biomass. J. Meteorol. Environ. 2006, $22,68-72$. 